\title{
A note on the ratio of the extreme to the root of the sum of squares in sequences of absolute values of Gaussian variables
}

\author{
Michiel A. Odijk \\ Independent Research, Gouda, The Netherlands
}

Pieter H.A.J.M. van Gelder

Faculty of Civil Engineering and Geosciences, Delft University of Technology, The Netherlands

\begin{abstract}
Let $Z_{1}, \ldots, Z_{n}$ be i.i.d. standard normal variables, $M_{n}$ the extreme among their absolute values, and $Q_{n}$ their rooted sum of squares. Here we study the ratio $v_{n}=M_{n} / Q_{n}$. We show that $\mu\left(v_{n}\right)=\mu\left(M_{n}\right) / \mu\left(Q_{n}\right) \sim b_{n} / \sqrt{n} \sim \sqrt{2 \log (n) / n}$, where $c_{n} \sim d_{n}$ denotes that $c_{n} / d_{n} \rightarrow 1$ for $\mathrm{n} \rightarrow \infty$. Moreover, convergence happens in a stable manner, i.e. $v_{n} \sim \mu\left(v_{n}\right)$ in probability. For low dimensions we also derive explicit expressions for $\mu\left(v_{n}\right)$ and variance $\sigma^{2}\left(v_{n}\right)$. These expressions are calculated by using an appealing geometrical interpretation of $v_{n}$.
\end{abstract}

\section{INTRODUCTION}

Let $\left\{Z_{n}\right\}$ be i.i.d. standard normal variables and define the entities

$$
\begin{aligned}
& M_{n}=\max _{i=1, \ldots, n}\left(\left|Z_{i}\right|\right), S_{n}=\sum_{i=1, \ldots, n}\left|Z_{i}\right|, Q_{n}=\sqrt{\sum_{i=1, \ldots, n} Z_{i}^{2}} \\
& \text { and } v_{n}=\frac{M_{n}}{Q_{n}} .
\end{aligned}
$$

For a random variable $X$, denote with $\mu(X)$ its mean and with $\sigma^{2}(X)$ its variance. If $\left\{p_{n}\right\}$ and $\left\{q_{n}\right\}$ are sequences, then $q_{n}=o\left(p_{n}\right)$ means that $q_{n} / p_{n} \rightarrow 0(n \rightarrow \infty)$. For notational convenience, when it is clear from the context, we don't mention explicitly that $n \rightarrow \infty$ when we discuss asymptotic behavior. Also, we write $p_{n} \sim q_{n}$ as a shorthand for $p_{n} / q_{n} \rightarrow 1$ or, equivalently, $p_{n}=q_{n}(1+o(1))$. Note that the tilde-operator defines an equivalence relation. Note that, if $p_{n} \sim q_{n}$ and $c_{n} \sim d_{n}$, then $p_{n} / c_{n} \sim q_{n} / d_{n}$.

As $Q_{n} \sim \sqrt{n}$ in probability (note that for all $\varepsilon>0$ Chebychev's inequality implies

$$
\begin{aligned}
& \operatorname{Pr}\left(\left|Q_{n} / \sqrt{n}-1\right|<\mathcal{E}\right) \\
& \left.=\operatorname{Pr}\left(\mid Q_{n}^{2} / n-1\right) /\left(Q_{n} / \sqrt{n}+1\right) \mid<\mathcal{E}\right) \\
& \quad \geq \operatorname{Pr}\left(\left|Q_{n}^{2} / n-1\right|<\mathcal{E}\right) \\
& \left.\quad \geq \sigma\left(\left|Z_{1}\right|^{2}\right) / \varepsilon \sqrt{n} \rightarrow 0\right)
\end{aligned}
$$

and $M_{n}$ is attracted by a stable law (meaning that there are sequences $\left\{a_{n}\right\}$ and $\left\{b_{n}\right\}$ such that $\left(M_{n}-b_{n}\right) / a_{n}$ converges in distribution to one of the extreme value distributions), it is tempting to believe that $v_{n}$ behaves asymptotically like $b_{n} / \sqrt{n}$. In this note we show that this intuition is indeed right and that in fact $\mu\left(v_{n}\right)=\mu\left(M_{n}\right) / \mu\left(Q_{n}\right) \sim$ $b_{n} / \sqrt{n} \sim \sqrt{2 \log (n) / n}$. Moreover, convergence of happens in a stable manner, i.e. $v_{n} \sim \mu\left(v_{n}\right)$ in probability.

A noteworthy result, that we use explicitly in this note, is that by Downey \& Wright (2007). They show that for non-negative i.i.d. random variables $X_{i}$ with finite second moment

$\mu\left(\frac{\max \left(X_{i}\right)}{\sum X_{i}}\right) \sim \frac{\mu\left(\max \left(X_{i}\right)\right)}{\mu\left(\sum X_{i}\right)}$.

In particular this implies that $\mu\left(M_{n} / S_{n}\right) \sim$ $\mu\left(M_{n}\right) / \mu\left(S_{n}\right)$, which mimicks $\mu\left(v_{n}\right)=\mu\left(M_{n}\right) /$ $\mu\left(Q_{n}\right)$, though in a weaker manner.

Central element to the above is the observation that $v_{n}$ has the same probability distribution as

$\eta_{n}:=\max \left\{\left|X_{1}\right|, \ldots,\left|X_{n}\right|\right\} \mid X=\left(X_{1}, \ldots, X_{n}\right) \sim U\left(B_{n}\right)$

where $\sim U\left(B_{n}\right)$ denotes sampling from the uniform distribution $U$ over $B_{n}$, being the surface of the n-dimensional unit hypersphere. This observation follows from the fact that points can be sampled randomly from $B_{n}$ by sampling from $\left(Z_{1}, \ldots, Z_{n}\right)$ and normalize the samples with $Q_{n}$ (see Muller 1959). In Section 2, this observation allows us to calculate $\mu\left(v_{n}\right)$ and $\sigma^{2}\left(v_{n}\right)$ explicitly for low values of $n$, which is another aim of this note. 
Also, by insensitivity of norms to scaling and translation, it follows that $v_{n}$ can be interpreted as the max-distance relative to the Euclidian-distance of two arbitrary points in n-space, which is an appealing geometrical interpretation of $v_{n}$. Put differently, the ratio $v_{n}$ can be interpreted as the longest edge of a hyperrectangle (also called orthotope), normalised by the space diagonal (which is $Q_{n}$ ).

In perspective, a vast amount of literature exists that deals with the properties of the ratio $M_{n} / S_{n}$. Application of this ratio can be found in the analysis of process speedup and the performance of scheduling (Downey \& Wright 2007), online bin-batching and wireless link quality (Zhang 2001; Nguyen 2011) or in the modelling of high dimensional databases (Brabants 2005). Like with $v_{n}=M_{n} / Q_{n}$, the ratio $M_{n} / S_{n}$ can be interpreted as the longest edge of a hyperrectangle, normalised by the sum of the lengths of its edges (which is $S_{n}$, taking one edge per dimension).

\section{EXACT RESULT}

In this section we calculate exact values for $\mu\left(v_{n}\right)$ and $\sigma^{2}\left(v_{n}\right)$ for low values of $n$. To do this, like mentioned in the introduction, we use the observation that $v_{n}$ and $\eta_{n}:=\max \left\{\left|X_{1}\right|, \ldots,\left|X_{n}\right|\right\} \mid X=\left(X_{1}, \ldots, X_{n}\right) \sim U\left(B_{n}\right)$ have the same distribution as a consequence of the fact that points can be sampled randomly from unit hypersphere $B_{n}$ by sampling from $Z_{1}, \ldots, Z_{n}$ and normalize the samples with $Q_{n}$.

Let $B_{n}^{*}:=\left\{x \in B_{n} \mid x_{1} \geq x_{2} \geq \cdots \geq x_{n} \geq 0\right\}$, i.e. $B_{n}^{*}$ is the part of $B_{n}$ of which all points are non-negative and define a non-increasing mapping. It is not difficult to see that by virtue of symmetry properties $\mu\left(\eta_{n}^{k}\right)=\mu\left(\eta_{n}^{k} \mid B_{n}^{*}\right)$, where $\mu\left(\eta_{n}^{k} \mid\right.$.) denotes the conditional $k$-th moment of $\eta_{n}$. Note that $\operatorname{vol}\left(B_{n}^{*}\right)=\operatorname{vol}\left(B_{n}\right) / 2^{n} n$ !.

When integrating over $B_{n}^{*}$ we switch to polar coordinates. That is, if $x \in B_{n}^{*}$, then $\varphi=\left(\varphi_{1}, \ldots, \varphi_{n-1}\right) \in[0, \pi / 2]^{n-1}$ and

$x_{1}=\cos \left(\varphi_{1}\right) \cos \left(\varphi_{2}\right) \cdots \cos \left(\varphi_{n-1}\right)$

$x_{2}=\sin \left(\varphi_{1}\right) \cos \left(\varphi_{2}\right) \cdots \cos \left(\varphi_{n-1}\right)$

$x_{3}=\sin \left(\varphi_{2}\right) \cos \left(\varphi_{3}\right) \cdots \cos \left(\varphi_{n-1}\right)$

....

$x_{n-1}=\sin \left(\varphi_{n-2}\right) \cos \left(\varphi_{n-1}\right)$

$x_{n}=\sin \left(\varphi_{n-1}\right)$

the Jacobian of which is well known and satisfies

$J_{n}(\varphi)=J_{n}\left(\varphi_{1}, \ldots, \varphi_{n-1}\right)=\cos ^{n-2}\left(\varphi_{n-1}\right) \cdot J_{n-1}\left(\varphi_{1}, \ldots, \varphi_{n-2}\right)$

with $J_{2}(\varphi)=1$. In terms of polar coordinates we have
$B_{n}^{*}=\left\{x(\varphi) \in B_{n} \mid \tan \left(\varphi_{1}\right) \leq 1, \tan \left(\varphi_{2}\right) \leq \sin \left(\varphi_{1}\right)\right.$, $\left.\tan \left(\varphi_{3}\right) \leq \sin \left(\varphi_{2}\right), \ldots, \tan \left(\varphi_{n-1}\right) \leq \sin \left(\varphi_{n-2}\right)\right\}$.

Clearly $\max \left\{x_{1}, \ldots, x_{n}\right\}=x_{1}=\cos \left(\varphi_{1}\right) \cdots \cos \left(\varphi_{n-1}\right)$ for $x \in B_{n}^{*}$. Hence, the $k$-th moment of $v_{n}$ is calculated as

$$
\begin{aligned}
& \mu\left(v_{n}^{k}\right)=\mu\left(\eta_{n}^{k}\right)=\frac{2^{n} n !}{\operatorname{vol}\left(B_{n}\right)} \int_{\tan \left(\varphi_{1}\right) \leq 1} \\
& \ldots \quad \int_{\tan \left(\varphi_{n-1}\right) \leq \sin \left(\varphi_{n-2}\right)}\left(\cos \left(\varphi_{1}\right) \cdots \cos \left(\varphi_{n-1}\right)\right)^{k} J_{n}(\varphi) d \varphi_{n-1} \cdots d \varphi_{1} .
\end{aligned}
$$

Evaluating this integral is quite cumbersome for large values of $n$, even for $k=1$ or $k=2$ (the latter being required to calculate $\left.\sigma^{2}\left(v_{n}\right)\right)$. In Appendix A we derive for lower dimensions

$$
\begin{aligned}
& \text { n } \mu\left(v_{n}\right) \quad \text { approx. } \sigma^{2}\left(v_{n}\right) \quad \text { approx. } \\
& \begin{array}{lllll}
1 & 1 & 1 & 0 & 0
\end{array} \\
& \begin{array}{llll}
2 & 2 \sqrt{2} \\
\pi & 0.900 & \frac{1}{2}+\frac{1}{\pi}-\frac{8}{\pi^{2}} & 0.00774
\end{array}
\end{aligned}
$$

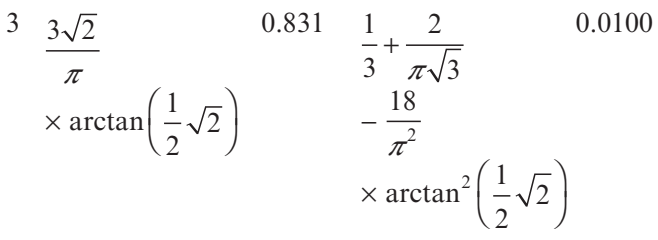

$$
\begin{aligned}
& 4 \quad 8 \sqrt{2} \quad 0.779 \\
& \begin{array}{l}
\frac{32 \sqrt{2}}{\pi^{2}} \\
-\frac{8 \sqrt{2}}{\pi}
\end{array} \\
& \times \arctan \left(\frac{1}{2} \sqrt{2}\right)
\end{aligned}
$$

For $n=2$ the probability distribution has support $[1 / \sqrt{2}, 1]$ and reads

$$
\begin{aligned}
F_{2}(z) & =\operatorname{Pr}\left(v_{2} \leq z\right)=\operatorname{Pr}\left(\cos \left(\varphi_{1}\right) \leq z \mid \varphi_{1} \sim U[0, \pi / 4]\right) \\
& =1-\frac{4}{\pi} \arccos (z)
\end{aligned}
$$

for $z$ in the support.

\section{MOMENT CONVERGENCE AND STABILITY OF $v_{n}$}

Following the previous section, since $\operatorname{vol}\left(B_{n}\right)=2 \pi^{n / 2} / \Gamma(n / 2), Q_{n}$ is chi-squared distributed with $n$ degrees of freedom and density $\mathrm{X}(t, n)$, and $M_{n}^{k}=\max \left\{\left|Z_{1}\right|^{k}, \ldots,\left|Z_{n}\right|^{k}\right\}$ we have 


$$
\begin{aligned}
& \mu\left(M_{n}^{k}\right)=2^{n} n ! \int_{\tan \left(\varphi_{1}\right) \leq 1} \cdots \int_{\tan \left(\varphi_{n-1}\right) \leq \sin \left(\varphi_{n-2}\right)} \\
& \int_{0}^{\infty}\left(r \cos \left(\varphi_{1}\right) \cdots \cos \left(\varphi_{n-1}\right)\right)^{k} \\
& \vartheta^{n}(r) J_{n}(\varphi) r^{n-1} d r d \varphi_{n-1} \cdots d \varphi_{1} \\
& =\frac{2^{n} n !}{\operatorname{vol}\left(B_{n}\right)} \int_{\tan \left(\varphi_{1}\right) \leq 1} \\
& \int\left(\cos \left(\varphi_{1}\right) \cdots \cos \left(\varphi_{n-1}\right)\right)^{k} J_{n}(\varphi) d \varphi_{n-1} \cdots d \varphi_{1} \times \cdots \\
& \tan \left(\varphi_{n-1}\right) \leq \sin \left(\varphi_{n-2}\right) \\
& \cdots \times \operatorname{vol}\left(B_{n}\right) \int_{0}^{\infty} \frac{1}{(2 \pi)^{n / 2}} e^{-r^{2} / 2} r^{n-1+k} d r \\
& =\mu\left(v_{n}^{k}\right) \operatorname{vol}\left(B_{n}\right) \int_{0}^{\infty} \frac{1}{(2 \pi)^{n / 2}} e^{-r^{2} / 2}\left(r^{2}\right)^{(n-2+k) / 2} d\left(r^{2} / 2\right) \\
& =\mu\left(v_{n}^{k}\right) \frac{\operatorname{vol}\left(B_{n}\right) \Gamma(n / 2)}{2 \pi^{n / 2}} \int_{0}^{\infty} t^{k / 2} \frac{1}{2^{n / 2} \Gamma(n / 2)} e^{-t / 2} t^{n / 2-1} d t \\
& =\mu\left(v_{n}^{k}\right) \int_{0}^{\infty} t^{k / 2} \mathrm{X}(t, n) d t \\
& =\mu\left(v_{n}^{k}\right) \mu\left(Q_{n}^{k}\right)
\end{aligned}
$$

where $\Gamma$ is the Gamma function, for which it is known that $\Gamma(k)=(k-1)$ ! and $\Gamma(k+1 / 2)=\sqrt{\pi}(2 k-1) ! ! / 2^{k}$.

Furthermore, it is well-known in literature that $\mu\left(M_{n}\right) \sim b_{n} \sim \sqrt{2 \log (n)}$, where $b_{n}$ is defined in the introduction and more precise in Appendix B. As to $\mu\left(Q_{n}\right)$, note that

$\mu\left(Q_{n}\right)=\int_{0}^{\infty} \sqrt{x} \frac{x^{n / 2-1} e^{-x / 2}}{2^{n / 2} \Gamma(n / 2)} d x=\sqrt{2} \frac{\Gamma((n+1) / 2)}{\Gamma(n / 2)}$

By observing that $\mu\left(Q_{n-1}\right)<\mu\left(Q_{n}\right)<\mu\left(Q_{n+1}\right)$, we calculate straightforwardly that $n-1=\mu\left(Q_{n}\right) \mu\left(Q_{n-1}\right)<\mu^{2}\left(Q_{n}\right)<\mu\left(Q_{n}\right) \mu\left(Q_{n+1}\right)=n$, so that $\sqrt{n-1}<\mu\left(Q_{n}\right)<\sqrt{n}$. Hence $\mu\left(Q_{n}\right) \sim \sqrt{n}$, implying

$\mu\left(v_{n}\right)=\frac{\mu\left(M_{n}\right)}{\mu\left(Q_{n}\right)} \sim \frac{b_{n}}{\sqrt{n}} \sim \sqrt{\frac{2 \log (n)}{n}}$.

Also, from Appendix it follows directly (note that $\left.\mu\left(Q_{n}^{2}\right)=n\right)$ that

$\sigma\left(v_{n}^{2}\right)-\frac{\pi^{2}}{6 n b_{n}^{2}} \rightarrow 0$

so that $\sigma\left(v_{n}^{2}\right) \rightarrow 0$.
From Appendix B it also follows straightforwardly that

$$
\begin{aligned}
\frac{\sigma^{2}\left(v_{n}\right)}{\mu^{2}\left(v_{n}\right)} & =\frac{\mu\left(v_{n}^{2}\right)}{\mu^{2}\left(v_{n}\right)}-1=\frac{\mu\left(M_{n}^{2}\right) / \mu\left(Q_{n}^{2}\right)}{\mu^{2}\left(M_{n}\right) / \mu^{2}\left(Q_{n}\right)}-1 \\
& =\frac{\left(b_{n}^{2} / n\right)(1+o(1))}{\left(b_{n} / \sqrt{n}\right)^{2}(1+o(1))}-1 \rightarrow 0
\end{aligned}
$$

and so (applying Chebychev's inequality), for all $\varepsilon>0$

$$
\begin{gathered}
\operatorname{Pr}\left(\left|\frac{v_{n}-\mu\left(v_{n}\right)}{\mu\left(v_{n}\right)}\right| \leq \varepsilon\right) \\
=\operatorname{Pr}\left(\left|v_{n}-\mu\left(v_{n}\right)\right| \leq \varepsilon \frac{\mu\left(v_{n}\right)}{\sigma\left(v_{n}\right)} \sigma\left(v_{n}\right)\right) \\
\geq 1-\frac{\sigma^{2}\left(v_{n}\right)}{\mu^{2}\left(v_{n}\right)} \frac{1}{\varepsilon^{2}} \rightarrow 1
\end{gathered}
$$

Thus, we conclude that $v_{n}$ is relatively stable, i.e. $v_{n} \sim \mu\left(v_{n}\right)$ in probability. Note that since $\mu\left(v_{n}\right) \rightarrow 0$ this is a stronger concept than absolute stability.

\section{SIMULATION OF $v_{n}$}

Following the previous elaboration, a Monte Carlo simulation is conducted to verify the asymptotic behaviour towards $\sqrt{2 \log (n) / n}$. The simulation code as included in Appendix $\mathrm{C}$ is used for this purpose. Figure 1 shows the result of 1000 runs. It is seen that the realisations are symmetrically distributed around the mean value and that the uncertainty vanishes large $n$.

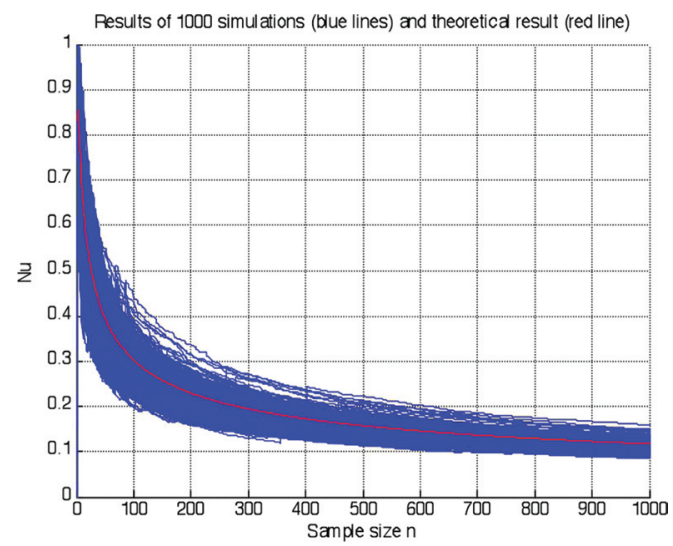

Figure 1. 


\section{REFERENCES}

Brabants, M. 2005, Introduction to Multimedia Databases, $\mathrm{PhD}$ thesis University of Hasselt, Belgium, (in Dutch).

Cramer, H.1946, Mathematical methods in statistics, Princeton University.

Deo, C.M. 1972, Some limit theorems for maxima of absolute values of Gaussian sequences, Indian Journal of Stat. (Series A) 34(3): 289-292.

Downey, P.J. \& Wright, P.E. 2007, The ratio of the extreme to the sum in a random sequence, Extremes 10(4): 249-266, Alternatively, see working paper TR 94-18 by the same authors and with the same title from the Dep. of Comp.Sc., University of Arizona, 1994.

Falk, M. \& Marohn, F. 1993, Von Mises conditions revisited, Ann. Prob. 21(3): 1310-1328.

Hoorfar, A. \& Hassani, M. 2008, Inequalities on the Lambert W function and hyperpower function, Journal of Inequalities in Pure and Appl. Math. 9(2), Article 51.
Muller, M.E. 1959, A note on a method for generating points uniformly on n-dimensional spheres, Communications of the ACM 2(4): 19-20.

Nair, K.A. 1981, Asymptotic distribution and moments of normal extremes, Ann. Prob. 9(1): 150-153.

Nguyen V.M. 2011, Wireless Link Quality Modelling and Mobility Management for Cellular Networks, Report Bell Labs and TREC Collaboration ( $s$.

Pickands III, J. 1968, Moment convergence of sample extremes, Ann. of Math. Stat. 39(3): 881-889.

Smith, R.L. 1987, Approximations in extreme value theory, Technical Report 205, Dept. Statistics, Univ. North Carolina.

von Mises, R. 1954, La distribution de la plus grande de n valeurs, Amer. Math. Soc., reprinted in: Selected Papers II: 271-294.

Zhang G. 2001, An on-line bin-batching problem, Discrete Appl. Math. 108(3): 329-333. 


\section{APPENDIX A: DERIVATIONS OF EXACT}

\section{RESULTS}

For $n=2$ we have

$\begin{aligned} \mu\left(v_{2}\right)= & \frac{2 ! 2^{2}}{\operatorname{vol}\left(B_{2}\right)} \int_{0}^{\pi / 4} \cos \left(\varphi_{1}\right) d \varphi_{1}=\frac{8}{2 \pi}\left(\frac{1}{2} \sqrt{2}\right)=\frac{2 \sqrt{2}}{\pi} \\ & \approx 0,900\end{aligned}$

Likewise, for $n=3$ we calculate

$$
\begin{aligned}
\mu\left(v_{3}\right)= & \frac{3 ! 2^{3}}{\operatorname{vol}\left(B_{3}\right)} \int_{0}^{\pi / 4} \int_{0}^{\arctan \left(\sin \left(\varphi_{1}\right)\right)} \cos \left(\varphi_{1}\right) \cdot \cos ^{2}\left(\varphi_{2}\right) d \varphi_{2} d \varphi_{1} \\
= & \frac{48}{4 \pi} \int_{0}^{\pi / 4} \cos \left(\varphi_{1}\right) \frac{1}{2}\left[\operatorname { s i n } \left(\arctan \left(\sin \left(\varphi_{1}\right)\right)\right.\right. \\
& +\cdots+\arctan \left(\sin \left(\varphi_{1}\right)\right) \\
= & \frac{6}{\pi} \int_{0}^{\pi / 4} \cos \left(\varphi_{1}\right)\left(\frac{1}{\sqrt{1+\sin \left(\varphi_{1}\right)}\left(\varphi_{1}\right)} \cdot \frac{\sin \left(\varphi_{1}\right)}{\sqrt{1+\sin ^{2}\left(\varphi_{1}\right)}}\right) d \varphi_{1} \\
= & \frac{6}{\pi} \int_{0}^{1 / \sqrt{2}} \frac{y}{1+y^{2}}+\arctan \left(\sin \left(\varphi_{1}\right)\right) \\
= & \frac{3}{\pi}[y \arctan (y)]_{0}^{1 / \sqrt{2}} \arctan \left(\frac{1}{2} \sqrt{2}\right) \approx 0,831 \\
& \frac{\arctan }{2}
\end{aligned}
$$

and for $n=4$

$$
\begin{aligned}
& \mu\left(v_{4}\right)=\frac{4 ! 2^{4}}{\operatorname{vol}\left(B_{4}\right)} . \\
& \int_{0}^{\pi / 4} \int_{0}^{\arctan \left(\sin \left(\varphi_{2}\right)\right)} \arctan \left(\sin \left(\varphi_{1}\right)\right) \\
& \int_{0}^{\arctan \left(\sin \left(\varphi_{2}\right)\right)} \cos \left(\varphi_{1}\right) \cos ^{2}\left(\varphi_{2}\right) \cos ^{3}\left(\varphi_{3}\right) d \varphi_{3} d \varphi_{2} d \varphi_{1} \\
& =\frac{384}{2 \pi^{2}} \int_{0}^{\pi / 4} \int_{0}^{\arctan \left(\sin \left(\varphi_{1}\right)\right)} \cos \left(\varphi_{1}\right) \cos ^{2}\left(\varphi_{2}\right) \frac{2}{3} \quad+\cdots \\
& \cdots+\frac{384}{2 \pi^{2}} \int_{0}^{\pi / 4} \int_{0}^{\arctan \left(\sin \left(\varphi_{1}\right)\right) \cos \left(\varphi_{1}\right) \cos ^{2}\left(\varphi_{2}\right)} \frac{1}{3} \sin \left(\arctan \left(\sin \left(\varphi_{2}\right)\right)\right) \cdots
\end{aligned}
$$

$$
\cdots \cos ^{2}\left(\arctan \left(\sin \left(\varphi_{2}\right)\right)\right) d \varphi_{2} d \varphi_{1}
$$

$$
\begin{aligned}
=\frac{128}{\pi^{2}} \int_{0}^{\pi / 4} & \int_{0}^{\arctan \left(\sin \left(\varphi_{1}\right)\right)} \cos \left(\varphi_{1}\right) \cos ^{2}\left(\varphi_{2}\right) \frac{\sin \left(\varphi_{2}\right)}{\sqrt{1+\sin ^{2}\left(\varphi_{2}\right)}} d \varphi_{2} d \varphi_{1}+\cdots
\end{aligned}
$$

$$
\cdots+\frac{64}{\pi^{2}} \int_{0}^{\pi / 4}
$$$$
\int_{0}^{\arctan \left(\sin \left(\varphi_{1}\right)\right)} \cos \left(\varphi_{1}\right) \cos ^{2}\left(\varphi_{2}\right) \frac{\sin \left(\varphi_{2}\right)}{\left(1+\sin ^{2}\left(\varphi_{2}\right)\right)^{3 / 2}} d \varphi_{2} d \varphi_{1}
$$$$
=\frac{128}{\pi^{2}} \int_{0}^{\pi / 4}
$$

$\arctan \left(\sin \left(\varphi_{1}\right)\right)$

$$
\int_{0}^{\infty} \cos \left(\varphi_{1}\right) \cos \left(\varphi_{2}\right) d \sqrt{1+\sin ^{2}\left(\varphi_{2}\right)} d \varphi_{1}+\cdots
$$

$$
\ldots+\frac{64}{\pi^{2}} \int_{0}^{\pi / 4}
$$

$$
\int_{0}^{\arctan \left(\sin \left(\varphi_{1}\right)\right)} \cos \left(\varphi_{1}\right) \cos \left(\varphi_{2}\right) \sin \left(\varphi_{2}\right) d \frac{\sin \left(\varphi_{2}\right)}{\sqrt{1+\sin ^{2}\left(\varphi_{2}\right)}} d \varphi_{1}
$$$$
=\frac{128}{\pi^{2}}\left[\int_{0}^{\pi / 4} \cos \left(\varphi_{1}\right) \frac{\sqrt{1+2 \sin ^{2}\left(\varphi_{1}\right)}}{1+\sin ^{2}\left(\varphi_{1}\right)} d \varphi_{1}-\frac{1}{2} \sqrt{2}\right]-\cdots
$$$$
\cdots-\frac{128}{\pi^{2}}\left[\int_{0}^{\pi / 4} \cos \left(\varphi_{1}\right) \int_{0}^{\arctan \left(\sin \left(\varphi_{1}\right)\right)} \sqrt{2-\cos ^{2}\left(\varphi_{2}\right)} d \cos \left(\varphi_{2}\right) d \varphi_{1}\right]
$$$$
+\cdots
$$

$$
\cdots+\frac{64}{\pi^{2}} \int_{0}^{\pi / 4} \cos \left(\varphi_{1}\right)\left[\begin{array}{l}
\frac{\sin ^{2}\left(\varphi_{1}\right)}{\left(1+\sin ^{2}\left(\varphi_{1}\right)\right) \sqrt{1+2 \sin ^{2}\left(\varphi_{1}\right)}} \\
-\int_{1 / \sqrt{1+\sin ^{2}\left(\varphi_{1}\right)}}^{1} \frac{2 y^{2}-1}{\sqrt{2-y^{2}}} d y
\end{array}\right] d \varphi_{1}
$$

$=\frac{128}{\pi^{2}} A+\frac{64}{\pi^{2}} B$.

where

$$
\begin{aligned}
A= & \int_{0}^{1 / \sqrt{2}} \frac{\sqrt{1+2 y^{2}}}{1+y^{2}} d y-\frac{1}{2} \sqrt{2} \\
& +\int_{0}^{\pi / 4} \cos \left(\varphi_{1}\right) \int_{1 / \sqrt{1+\sin ^{2}\left(\varphi_{1}\right)}}^{1} \sqrt{2-y^{2}} d y d \varphi_{1}
\end{aligned}
$$


and

$$
\begin{aligned}
B= & \int_{0}^{\pi / 4} \cos \left(\varphi_{1}\right)\left[\begin{array}{c}
\frac{\sin ^{2}\left(\varphi_{1}\right)}{\left(1+\sin ^{2}\left(\varphi_{1}\right)\right) \sqrt{1+2 \sin ^{2}\left(\varphi_{1}\right)}} \\
\int_{1 / \sqrt{1+\sin ^{2}\left(\varphi_{1}\right)}}^{1} \frac{2 y^{2}-1}{\sqrt{2-y^{2}}} d y
\end{array}\right] d \varphi_{1} \\
= & \int_{0}^{1 / \sqrt{2}} \frac{y^{2}}{\left(1+y^{2}\right) \sqrt{1+2 y^{2}}} d y \\
& -\int_{0}^{\pi / 4} \cos \left(\varphi_{1}\right) \int_{1 / \sqrt{1+\sin ^{2}\left(\varphi_{1}\right)}}^{1} \frac{2 y^{2}-1}{\sqrt{2-y^{2}}} d y d \varphi_{1}
\end{aligned}
$$

Since

$$
\begin{aligned}
& \int_{1 / \sqrt{1+\sin ^{2}\left(\varphi_{1}\right)}}^{1} \sqrt{2-y^{2}} d y \\
& =\left[\frac{1}{2} y \sqrt{2-y^{2}}+\arcsin \left(\frac{1}{2} \sqrt{2} y\right)\right]_{1 / \sqrt{1+\sin ^{2}\left(\varphi_{1}\right)}}^{1} \\
& =\frac{1}{2}+\frac{1}{4} \pi-\frac{\sqrt{1+2 \sin ^{2}\left(\varphi_{1}\right)}}{2+2 \sin ^{2}\left(\varphi_{1}\right)}-\arcsin \left(\frac{1}{\sqrt{2+2 \sin ^{2}\left(\varphi_{1}\right)}}\right)
\end{aligned}
$$

we find

$$
\begin{gathered}
\int_{0}^{\pi / 4} \cos \left(\varphi_{1}\right) \int_{1 / \sqrt{1+\sin ^{2}\left(\varphi_{1}\right)}}^{1} \sqrt{2-y^{2}} d y d \varphi_{1}=\frac{1}{4} \sqrt{2}+\frac{1}{8} \pi \sqrt{2} \\
-\int_{0}^{1 / \sqrt{2}} \frac{\sqrt{1+2 y^{2}}}{2+2 y^{2}} d y-\int_{0}^{1 / \sqrt{2}} \arcsin \left(\frac{1}{\sqrt{2+2 y^{2}}}\right) d y .
\end{gathered}
$$

Also, we have

$$
\begin{aligned}
& \int_{0}^{1 / \sqrt{2}} \arcsin \left(\frac{1}{\sqrt{2+2 y^{2}}}\right) d y=\left[y \arcsin \left(\frac{1}{\sqrt{2+2 y^{2}}}\right)\right]_{0}^{1 / \sqrt{2}} \\
& +\int_{0}^{1 / \sqrt{2}} \frac{2 y^{2}}{\left(2+2 y^{2}\right) \sqrt{1+2 y^{2}}} d y \\
& =\frac{1}{2} \sqrt{2} \arcsin \left(\frac{1}{3} \sqrt{3}\right)+\int_{0}^{1 / \sqrt{2}} \frac{2 y^{2}}{\left(2+2 y^{2}\right) \sqrt{1+2 y^{2}}} d y
\end{aligned}
$$

so that

$$
\begin{aligned}
A= & -\frac{1}{4} \sqrt{2}+\frac{1}{8} \pi \sqrt{2}-\frac{1}{2} \sqrt{2} \arcsin \left(\frac{1}{3} \sqrt{3}\right) \\
& +\int_{0}^{1 / \sqrt{2}} \frac{1}{\left(2+2 y^{2}\right) \sqrt{1+2 y^{2}}} d y
\end{aligned} .
$$

Furthermore,

$$
\begin{aligned}
& \int_{1 / \sqrt{1+\sin ^{2}\left(\varphi_{1}\right)}}^{1} \frac{2 y^{2}-1}{\sqrt{2-y^{2}}} d y=\int_{1 / \sqrt{1+\sin ^{2}\left(\varphi_{1}\right)}}^{1} \frac{2 y^{2}-4}{\sqrt{2-y^{2}}} d y \\
& +\int_{1 / \sqrt{1+\sin ^{2}\left(\varphi_{1}\right)}}^{1} \frac{3}{\sqrt{2-y^{2}}} d y \\
& =-2 \int_{1 / \sqrt{1+\sin ^{2}\left(\varphi_{1}\right)}}^{\sqrt{2-y^{2}} d y+3} \int_{1 / \sqrt{1+\sin ^{2}\left(\varphi_{1}\right)}}^{1} \frac{1}{\sqrt{2-y^{2}}} d y \\
& =-2\left[\frac{1}{2}+\frac{1}{4} \pi-\frac{\sqrt{1+2 \sin ^{2}\left(\varphi_{1}\right)}}{2+2 \sin ^{2}\left(\varphi_{1}\right)}\right. \\
& \left.-\arcsin \left(\frac{1}{\sqrt{2+2 \sin ^{2}\left(\varphi_{1}\right)}}\right)\right]+\cdots \\
& =-1+\frac{1}{4} \pi+\frac{\sqrt{1+2 \sin ^{2}\left(\varphi_{1}\right)}}{1+\sin ^{2}\left(\varphi_{1}\right)} \\
& -\arcsin \left(\frac{1}{\sqrt{2+2 \sin ^{2}\left(\varphi_{1}\right)}}\right)
\end{aligned}
$$

so that

$$
\begin{aligned}
& B=\int_{0}^{1 / \sqrt{2}} \frac{\left(2 y^{2}+1\right)-\left(1+y^{2}\right)}{\left(1+y^{2}\right) \sqrt{1+2 y^{2}}} d y-\cdots \\
& \cdots-\int_{0}^{\pi / 4}\left[\begin{array}{l}
-1+\frac{1}{4} \pi+\frac{\sqrt{1+2 \sin ^{2}\left(\varphi_{1}\right)}}{1+\sin ^{2}\left(\varphi_{1}\right)} \\
-\arcsin \left(\frac{1}{\sqrt{2+2 \sin ^{2}\left(\varphi_{1}\right)}}\right)
\end{array}\right] d \sin \left(\varphi_{1}\right) \\
& =\int_{0}^{1 / \sqrt{2}} \frac{\left(2 y^{2}+1\right)-\left(1+y^{2}\right)}{\left(1+y^{2}\right) \sqrt{1+2 y^{2}}} d y \\
& -\int_{0}^{1 / \sqrt{2}}\left[-1+\frac{1}{4} \pi+\frac{\sqrt{1+2 y^{2}}}{1+y^{2}}-\arcsin \left(\frac{1}{\sqrt{2+2 y^{2}}}\right)\right] d y \\
& =\int_{0}^{1 / \sqrt{2}} \frac{-1}{\sqrt{1+2 y^{2}}} d y \\
& +\int_{0}^{1 / \sqrt{2}}\left[1-\frac{1}{4} \pi+\arcsin \left(\frac{1}{\sqrt{2+2 y^{2}}}\right)\right] d y
\end{aligned}
$$

$$
\begin{aligned}
= & \int_{0}^{1 / \sqrt{2}} \frac{-1}{\sqrt{1+2 y^{2}}} d y+\frac{1}{2} \sqrt{2}-\frac{1}{8} \pi \sqrt{2}+\frac{1}{2} \sqrt{2} \arcsin \left(\frac{1}{3} \sqrt{3}\right) \\
& +\int_{0}^{1 / \sqrt{2}} \frac{2 y^{2}}{\left(2+2 y^{2}\right) \sqrt{1+2 y^{2}}} d y
\end{aligned}
$$




$$
\begin{aligned}
= & \frac{1}{2} \sqrt{2}-\frac{1}{8} \pi \sqrt{2}+\frac{1}{2} \sqrt{2} \arcsin \left(\frac{1}{3} \sqrt{3}\right) \\
& +\int_{0}^{1 / \sqrt{2}} \frac{-2}{\left(2+2 y^{2}\right) \sqrt{1+2 y^{2}}} d y
\end{aligned}
$$

Hence

$$
\begin{aligned}
& \mu\left(v_{4}\right) \\
& =\frac{128}{\pi^{2}}\left[\begin{array}{l}
-\frac{1}{4} \sqrt{2}+\frac{1}{8} \pi \sqrt{2}-\frac{1}{2} \sqrt{2} \arcsin \left(\frac{1}{3} \sqrt{3}\right) \\
+\int_{0}^{1 / \sqrt{2}} \frac{1}{\left(2+2 y^{2}\right) \sqrt{1+2 y^{2}}} d y
\end{array}\right]+\cdots \\
& \cdots+\frac{64}{\pi^{2}}\left[\begin{array}{l}
\frac{1}{2} \sqrt{2}-\frac{1}{8} \pi \sqrt{2}+\frac{1}{2} \sqrt{2} \arcsin \left(\frac{1}{3} \sqrt{3}\right) \\
+\int_{0}^{1 / \sqrt{2}} \frac{-2}{\left(2+2 y^{2}\right) \sqrt{1+2 y^{2}}} d y
\end{array}\right] \\
& =\frac{8 \sqrt{2}}{\pi}-\frac{32 \sqrt{2}}{\pi^{2}} \arcsin \left(\frac{1}{3} \sqrt{3}\right) \\
& =\frac{8 \sqrt{2}}{\pi}-\frac{32 \sqrt{2}}{\pi^{2}} \arctan \left(\frac{1}{2} \sqrt{2}\right) \\
& \approx 0.779
\end{aligned}
$$

As to the variance $\sigma^{2}\left(v_{2}\right)$ we calculate the second moment of $\mu_{2}\left(v_{2}\right)$ of $v_{2}$ as

$$
\begin{aligned}
\mu\left(v_{2}^{2}\right) & =\frac{2 ! 2^{2}}{\operatorname{vol}\left(B_{2}\right)} \int_{0}^{\pi / 4} \cos ^{2}\left(\varphi_{1}\right) d \varphi_{1} \\
& =\frac{8}{2 \pi}\left(\frac{1}{4}+\frac{\pi}{8}\right)=\frac{1}{\pi}+\frac{1}{2} \approx 0,818
\end{aligned}
$$

so that

$$
\sigma^{2}\left(v_{2}\right)=\frac{1}{\pi}+\frac{1}{2}-\frac{8}{\pi^{2}} \approx 0,00774
$$

Also

$$
\begin{aligned}
& \mu\left(v_{3}^{2}\right)=\frac{3 ! 2^{3}}{\operatorname{vol}\left(B_{3}\right)} \int_{0}^{\pi / 4} \arctan \left(\sin \left(\varphi_{1}\right)\right) \\
& \int_{0}^{2} \cos ^{2}\left(\varphi_{1}\right) \cdot \cos ^{3}\left(\varphi_{2}\right) d \varphi_{2} d \varphi_{1} \\
& =\frac{48}{4 \pi} \int_{0}^{\pi / 4} \cos ^{2}\left(\varphi_{1}\right)\left(\begin{array}{l}
\sin \left(\arctan \left(\sin \left(\varphi_{1}\right)\right)\right) \\
-\frac{1}{3} \sin ^{3}\left(\arctan \left(\sin \left(\varphi_{1}\right)\right)\right)
\end{array}\right) d \varphi_{1}
\end{aligned}
$$

$$
\begin{aligned}
= & \frac{12}{\pi} \int_{0}^{\pi / 4} \cos ^{2}\left(\varphi_{1}\right)\left(\begin{array}{c}
\frac{\sin \left(\varphi_{1}\right)}{\sqrt{1+\sin ^{2}\left(\varphi_{1}\right)}} \\
\left.-\frac{\sin ^{3}\left(\varphi_{1}\right)}{3\left(1+\sin ^{2}\left(\varphi_{1}\right)\right)^{3 / 2}}\right)
\end{array}\right) d \varphi_{1} \\
= & -\frac{12}{\pi} \int_{0}^{\pi / 4} \frac{\cos ^{2}\left(\varphi_{1}\right)}{\sqrt{2-\cos ^{2}\left(\varphi_{1}\right)} d \cos \left(\varphi_{1}\right)+\cdots} \\
& \cdots+\frac{12}{\pi} \int_{0}^{\pi / 4} \frac{\cos ^{2}\left(\varphi_{1}\right)\left(1-\cos ^{2}\left(\varphi_{1}\right)\right)}{3\left(2-\cos ^{2}\left(\varphi_{1}\right)\right)^{3 / 2}} d \cos \left(\varphi_{1}\right) \\
= & \frac{12}{\pi} \int_{1 / \sqrt{2}}^{1} \frac{y^{2}}{\sqrt{2-y^{2}}} d y-\frac{4}{\pi} \int_{1 / \sqrt{2}}^{1} \frac{y^{2}}{\left(2-y^{2}\right)^{3 / 2}} d y \\
& +\frac{4}{\pi} \int_{1 / \sqrt{2}}^{1} \frac{y^{4}}{\left(2-y^{2}\right)^{3 / 2}} d y \\
= & \frac{12}{\pi}\left[\arcsin \left(\frac{y}{\sqrt{2}}\right)-\frac{1}{2} y \sqrt{2-y^{2}}\right]_{1 / \sqrt{2}}^{1}-\cdots \\
& \cdots-\frac{4}{\pi}\left[\frac{y}{\sqrt{2-y^{2}}}-\arcsin \left(\frac{y}{\sqrt{2}}\right)\right]_{1 / \sqrt{2}}^{1}+\cdots \\
= & \frac{4}{\pi}\left[\arcsin \left(\frac{y}{\sqrt{2}}\right)-y \sqrt{2-y^{2}}+\frac{y}{\sqrt{2-y^{2}}}\right]_{1 / \sqrt{2}}^{1} \\
& \cdots+\frac{4}{\pi}\left[\frac{1}{2} y \sqrt{2-y^{2}}+\frac{2 y}{\sqrt{2-y^{2}}}-3 \arcsin \left(\frac{y}{\sqrt{2}}\right)\right]_{1 / \sqrt{2}}^{1} \\
& =1
\end{aligned}
$$

so that

$$
\sigma^{2}\left(v_{3}\right)=\frac{1}{3}+\frac{2}{\pi \sqrt{3}}-\frac{18}{\pi^{2}} \arctan ^{2}\left(\frac{1}{2} \sqrt{2}\right) \approx 0.0100 .
$$

\section{APPENDIX B: PROOF THAT,} $\mu\left(M_{n}\right) \sim b_{n}, \mu\left(M_{n}^{2}\right) \sim b_{n}^{2}$ AND $b_{n} \sim \sqrt{2 \log (n))}$

Let $\left\{Z_{n}\right\}$ and $Z$ be i.i.d. standard normal variables (with distribution $\Phi$ and density $\vartheta$ ). Let $\Phi_{a b s}(x)=\operatorname{Pr}(|Z| \leq x)$ and denote its density with $\vartheta_{a b s}$. Also, we write $\bar{\Phi}$ and $\bar{\Phi}_{a b s}$ as a shorthand for tail probabilities $1-\Phi$ and $1-\Phi_{a b s}$ respectively. Obviously, for $x \geq 0$ we have $\vartheta_{a b s}(x)=2 \vartheta(x)$ and $\bar{\Phi}_{a b s}(x)=2 \bar{\Phi}(x)=\operatorname{erfc}(x / \sqrt{2})$, where erfc is the complementary error function defined by and having first order Laurent expansion

$\operatorname{erfc}(x):=\frac{2}{\sqrt{\pi}} \int_{x}^{\infty} e^{-t^{2}} d t=\frac{e^{-x^{2}}}{x \sqrt{\pi}}(1+o(1))$. 
For the moments of $|Z|$ we have

$$
\begin{aligned}
\mu\left(|Z|^{k}\right) & =\sqrt{\frac{2}{\pi}} \int_{0}^{\infty} t^{k} e^{-t^{2} / 2} d t=-\sqrt{\frac{2}{\pi}} \int_{0}^{\infty} t^{k-1} d e^{-t^{2} / 2} \\
& =(k-1) \sqrt{\frac{2}{\pi}} \int_{0}^{\infty} t^{k-2} e^{-t^{2} / 2} d t=(k-1) \mu\left(|Z|^{k-2}\right)
\end{aligned}
$$

so that $\mu\left(|Z|^{2 i}\right)=(2 i-1) ! !$ and $\mu\left(|Z|^{2 i+1}\right)=$ $\sqrt{2 / \pi}(2 i)$ !! . Notably, moments exist and are finite. Also, the inverse $\bar{\Phi}_{a b s}^{\leftarrow}$ of $\bar{\Phi}_{a b s}$ will be used below and follows from

$$
\frac{1}{\bar{\Phi}_{a b s}^{2}(x)}=\frac{1}{e r f c^{2}(x / \sqrt{2})}=\frac{\pi}{2} x^{2} e^{x^{2}} \frac{1}{(1+o(1))^{2}}
$$

yielding

$$
\bar{\Phi}_{a b s}^{\leftarrow}(x)=\sqrt{W\left(\frac{2}{\pi x^{2}}(1+o(1))\right)}
$$

where $W$ is the Lambert $W$ function, implicitly defined by $W(x) e^{W(x)}=x$.

With $\Lambda$ we refer to the Gumbel distribution (also known as the doubly exponential distribution and being one of the three limiting cases of the GEV distribution, well known in extreme value theory) defined by $\Lambda(x)=\exp \left(-e^{-x}\right)$, having mean $\gamma$ and variance $\pi^{2} / 6$, where $\gamma \approx 0.577$ is the Euler-Mascheroni constant. It is common knowledge that $\Phi$ satisfies the von Mises condition for being in the domain of attraction of $\Lambda$ (von Mises 1936; Falk 1993), i.e.

$$
\frac{\vartheta(x)}{\bar{\Phi}^{2}(x)} \int_{x}^{\infty} \bar{\Phi}(t) d t \rightarrow 1 \quad(x \rightarrow \infty) .
$$

It is easily seen that $\Phi_{a b s}$ also satisfies this condition and is therefore also in the domain of attraction of $\Lambda$. Say that $\left\{a_{n}\right\}$ and $\left\{b_{n}\right\}$ are sequences for which $\left(M_{n}-b_{n}\right) / a_{n} \rightarrow \Lambda$ in distribution. Intuitively we would expect that $\mu\left(M_{n}\right)-\left(b_{n}+a_{n} \gamma\right) \rightarrow 0$ and that $\mu\left(M_{n}^{2}\right)-\left(a_{n}^{2}\left(\pi^{2} / 6+\gamma^{2}\right)+b_{n}^{2}+2 a_{n} b_{n} \gamma\right) \rightarrow 0$. From Pickands (1968) (see also Nair 1981) we know that this is indeed the case. Furthermore, it is widely known that $\left\{a_{n}\right\}$ and $\left\{b_{n}\right\}$ are solutions to $b_{n}=\bar{\Phi}_{a b s}^{\leftarrow}(1 / n)$ and $a_{n}=r\left(b_{n}\right)$, where $r(x)=\bar{\Phi}_{a b s}(x) / \vartheta_{a b s}(x)$ is the reciprocal hazard function (Smith 1987). Hence

$$
b_{n}=\sqrt{W\left(\frac{2 n^{2}}{\pi}(1+o(1))\right)}
$$

and

$$
a_{n}=\frac{1 / n}{\sqrt{2 / \pi} e^{-b_{n}^{2} / 2}}=\frac{1}{b_{n}} \sqrt{\frac{\pi}{2 n^{2}} b_{n}^{2} e^{b_{n}^{2}}} \sim \frac{1}{b_{n}} .
$$

Hence (note that $\mu\left(M_{n}\right)=b_{n}+o(1)$ and $b_{n} \rightarrow \infty$ ), $\mu\left(M_{n}\right) \sim b_{n}$ and $\mu\left(M_{n}^{2}\right) \sim b_{n}^{2}$.

Finally, as $\log (x)-\log \log (x) \leq W(x) \leq$ $\log (x)-\log \log (x) / 2$ for $x \geq e$ (see Hoorfar 2008), we see that in terms of elementary functions $b_{n} \sim \sqrt{2 \log (n)}$. Consistently, Cramer (1946) and Deo (1972) approximate the location and scaling parameters $\left\{a_{n}\right\}$ and $\left\{b_{n}\right\}$ by $b_{n}=c_{n}-\log (4 \pi \log (n)) / 2 c_{n}+2 / c_{n}$ and $a_{n}=1 / c_{n}$, where $c_{n}=\sqrt{2 \log (n)}$.

\section{APPENDIX C: MATLAB CODE FOR SIMULATION OF $v_{n}$}

Clear

$\mathrm{clg}$

$\mathrm{n}=1000$;

hold on

for $\mathrm{j}=1: \mathrm{n}$,

$\mathrm{z}(1)=\operatorname{normrnd}(0,1)$;

$\mathrm{m}(1)=\operatorname{abs}(\mathrm{z}(1))$;

$\mathrm{s}(1)=\mathrm{m}(1)$;

$\mathrm{q} 2(1)=\mathrm{z}(1)^{\wedge} 2$;

for $i=2: n$,

$z(i)=\operatorname{normrnd}(0,1)$;

$\mathrm{m}(\mathrm{i})=\max (\mathrm{m}(\mathrm{i}-1), \operatorname{abs}(\mathrm{z}(\mathrm{i})))$;

$\mathrm{s}(\mathrm{i})=\mathrm{s}(\mathrm{i}-1)+\mathrm{abs}(\mathrm{z}(\mathrm{i}))$;

$\mathrm{q} 2(\mathrm{i})=\mathrm{q} 2(\mathrm{i}-1)+\mathrm{z}(\mathrm{i})^{\wedge} 2$;

$q(i)=\operatorname{sqrt}(q 2(i))$;

nu(i) $=m(i) / q(i)$;

end

ex(i) $=\operatorname{sqrt}(2 * \log (i) / \log (2.7181) / i)$;

$\log \log \left([1: \mathrm{n}], \mathrm{nu},{ }^{\prime} \mathrm{b}^{\prime}\right)$

end

$\log \log \left([2: n], \operatorname{ex}(2: n), r^{\prime}\right)$

xlabel('Sample size $\left.\mathrm{n}^{\prime}\right)$

ylabel(' $\left.\mathrm{Nu}^{\prime}\right)$

grid

title('Results of 1000 simulations (blue lines) and theoretical result (red line)')

holf off 